\title{
Long-Term Follow-Up of Polypoidal Choroidal Vasculopathy Secondary to Angioid Streaks Treated by Intravitreal Aflibercept and Ranibizumab
}

\author{
James G. Wong Katherine Yu Qian \\ Strathfield Retina Clinic, Sydney, NSW, Australia; Medical Retina Unit, Sydney Eye \\ Hospital, Sydney, NSW, Australia; Save Sight Institute, University of Sydney, \\ Sydney, NSW, Australia
}

\section{Keywords}

Angioid streaks · Polypoidal choroidal vasculopathy · Choroidal neovascularisation · Choroidal nevus · Fluorescein angiography · Indocyanine green angiography · Optical coherence tomography $\cdot$ Subretinal fluid $\cdot$ Aflibercept $\cdot$ Ranibizumab

\begin{abstract}
Purpose: Angioid streaks (AS) are dehiscences in Bruch's membrane that may be idiopathic or associated with numerous systemic illnesses. Polypoidal choroidal vasculopathy (PCV) is an underdiagnosed exudative chorioretinopathy often characterised by serosanguineous detachments of the pigmented epithelium. The use of the anti-VEGF agents ranibizumab and aflibercept in the management of PCV secondary to AS has not been previously documented. We report 3 patients with active PCV secondary to AS, 1 of which had a family history of $\mathrm{PCV}$ secondary to AS, not previously reported in the literature. All patients were symptomatic and treated with intravitreal anti-VEGF therapy with and without combination photodynamic therapy (PDT). Methods: This is a long-term retrospective case review of 3 eyes of 3 patients with AS and clinical features of PCV. The patients were examined using fundoscopy, spectral domain optical coherence tomography, fluorescein angiography, and indocyanine green angiography. All patients were managed with intravitreal anti-VEGF using a treat-and-extend protocol according to specific retreatment criteria. One patient had 1 session of PDT in com-
\end{abstract}




\section{Case Reports in Ophthalmology}

bination with anti-VEGF injections. Results: The mean follow-up time in all patients was 4 years. In all 3 cases, the treatment resulted in improved visual acuity and regression of active PCV lesions with a longer duration between injections. Conclusion: The treat-and-extend protocol with intravitreal aflibercept or ranibizumab with or without PDT is effective and safe for PCV secondary to AS.

(C) 2017 The Author(s)

Published by S. Karger AG, Basel

\section{Introduction}

Angioid streaks (AS) are narrow, irregularly radiating lines emanating from the peripapillary area, resulting from pathological dehiscences in Bruch's membrane [1]. While they may be idiopathic, they are often associated with systemic conditions, most commonly pseudoxanthoma elasticum (PXE), Paget disease, and sickle haemoglobinopathies [2]. While generally asymptomatic, secondary complications may arise from choroidal neovascularisation (CNV), subretinal haemorrhage, and retinal pigment epithelial detachments (PED). Involvement of the macula may result in vision impairment and metamorphopsia $[3,4]$.

Polypoidal choroidal vasculopathy (PCV) is an exudative chorioretinopathy characterised by grape-like polypoidal subretinal vascular lesions associated with branching vascular networks (BVN) [5]. Controversy exists over whether PCV represents a subtype of neovascular age-related macular degeneration or a separate disease entity with different risk factors and outcomes. Typically, recurrent serous exudation and haemorrhage result in detachments of RPE which may cause fibrotic scarring, chorioretinal atrophy, and vision loss [6]. PCV can be difficult to distinguish from typical Type $1 \mathrm{CNV}$ in neovascular age-related macular degeneration, hence it is often underdiagnosed. Indocyanine green angiography (ICGA) is the gold standard for PCV diagnosis by highlighting typical polyp lesions and BVN within the choroidal vasculature.

Previous studies have demonstrated that the anti-VEGF agents ranibizumab and aflibercept are well-tolerated and efficacious treatments in the management of PCV [6]. However, their use in patients with PCV associated with AS has not been previously documented in the literature. We report the long-term follow-up of 3 clinical cases of PCV secondary to AS in which the patients were managed with intravitreal anti-VEGF injections with and without photodynamic therapy (PDT).

\section{Case Reports}

\section{Case 1}

A 52-year-old Caucasian man presented with a 2-week history of central distortion in his right eye. He had a family history of advanced PCV secondary to AS in his mother (Case 2) (Fig. 2). At initial presentation, visual acuity was 20/25 in the right eye and 20/30 in the left eye.

Dilated fundus examination of both eyes showed dark brown irregularly radiating linear lines emanating from the peripapillary area including through the macula, corresponding to the characteristic clinical appearance of AS (Fig. 1e, f). PEDs and subretinal haemorrhage adjacent to the area of AS in the right macula were noted clinically and on spectral domain optical coherence tomography examination (SD-OCT; Topcon Corporation, Tokyo, Japan). There were no other fundus features suggestive of PXE such as peau d'orange or comet lesions. There were no other related systemic clinical findings including skin lesions sugges- 
tive of any connective tissue disease. The patient was reviewed by a dermatologist and physician who did not find any obvious associated systemic disorders.

Fluorescein angiography (FA) demonstrated an occult (Type 1) choroidal neovascular membrane, and ICGA demonstrated grape-like choroidal vascular lesions consistent with a diagnosis of PCV. Intravitreal ranibizumab injections were commenced at 4 weekly intervals according to a treat-and-extend protocol. Following the stability of visual acuity and OCT parameters, the interval between injections was increased by 2 weeks to a maximum of 12 weeks. Retreatment criteria and the next treatment interval were based on evidence of disease activity including: loss of visual acuity of more than 5 ETDRS letters, presence of new haemorrhage, increased subretinal, intraretinal, or sub-RPE fluid. Subretinal haemorrhage was absent at 5 months; however, a small but persisting PED was observed, and the condition remained stable at 2 years with good vision.

However, at 3 years following commencement of treatment, whilst the treatment interval was at 12 weeks, the patient became symptomatic of a paracentral scotoma. Clinical examination revealed a large subretinal haemorrhage, subretinal fluid (SRF), and PED in the inferior macula (Fig. 1a, b).

FA (Fig. 1c, d) and ICGA (Fig. 1e, f) demonstrated early filling of a grape-like polypoidal structure with leakage in the late phase suggestive of PCV secondary to AS. ICGA also revealed an associated BVN in both the early and late phase (Fig. 1e, f). The patient received a combination of intravitreal ranibizumab with full fluence Visudyne PDT, and the injection treatment interval was reduced to 4 weeks until the lesion stabilised. Following this, further ranibizumab injections were administered according to the indicated treat-and-extend protocol. At 56 months, visual acuity stabilised to 20/20 in the right eye. Figure 2 demonstrates an improved OCT after the treatment and a repeat ICGA showing regressed PCV. In total, 19 ranibizumab injections were administered with a mean interval between consecutive injections of 89 days.

Fifty-seven months following the initiation of this treatment, the patient was receiving injections every 6 weeks, then he switched from ranibizumab to intravitreal aflibercept on a similar treat-and-extend protocol according to the same retreatment criteria.

Eight injections of aflibercept were subsequently applied during a 27-month follow-up period, with a mean of 85 consecutive days between aflibercept injections. At the final follow-up (84 months), visual acuity was 20/20 and OCT revealed an absence of SRF; however, a small but persisting PED (Fig. $1 \mathrm{~m}$ ). Over 84 months, a total number of 27 injections (19 ranibizumab and 8 aflibercept) were given in the right eye with an average of 90 days between injections (both types). In the first year, the injection interval was 31 days on average with ranibizumab, and 62 days in the 4 th year with aflibercept.

Case 2

An 84-year-old Caucasian woman (mother of Case 1) presented with bilateral, longstanding, poor vision since the age of 47 years. She had a history of controlled hypertension, atrial fibrillation, and hydrocephalus; however, no other known systemic disease associated with AS. Her visual acuity was 20/400 in the right and 20/200 in the left eye, and she had bilateral, hypertrophic, disciform, macular scars and evidence of AS. OCT revealed significant subretinal fibrosis; however, no significant SRF or intraretinal fluid. FA and ICGA were performed and demonstrated bilateral PCV with polyp lesions and BVN (Fig. 2). Due to her poor vision and established fibrotic macular scars, treatment was conservative. 


\section{Case Reports in Ophthalmology}

Case 3

A 72-year-old Caucasian woman with hypertension and hypercholesterolemia presented with a central scotoma in her left eye. Her best corrected visual acuity was 20/50 in the right eye and 20/50 in the left eye.

Fundoscopy showed irregular lines with a brownish appearance emanating from the optic disc and spreading radially in various directions in both eyes, corresponding to the clinical appearance of AS. There was no evidence of peau d'orange or other peripheral retinal abnormalities. In the left macula, OCT, FA, and ICGA examination demonstrated SRF, intraretinal fluid, and a Type 1 choroidal neovascular membrane without any evidence of PCV at this stage. There was no CNV in the right eye at this stage. A diagnosis of CNV secondary to AS in the left eye was made, and intravitreal ranibizumab injections were commenced according to a treat-and-inject protocol utilizing OCT and visual acuity criteria as outlined above for the first 2 years. This was subsequently switched to a PRN protocol. A total of 14 ranibizumab injections were given from baseline to 24 months, then no injections were given until 46 months. Vision improved to 20/25. She was reviewed by a dermatologist and physician who found no other systemic illnesses associated with AS.

At 46 months' follow-up of the left eye, the patient experienced new symptoms of distortion in the previously unaffected right eye. Clinical examination revealed a discreet orangered nodule in the nasal right macula adjacent to the AS (Fig. 3a). OCT revealed typical features of PCV, including a PED with an underlying polypoidal lesion (Fig. 3b). FA (Fig. 3e, f) and ICGA (Fig. 3g, h) demonstrated a polypoidal lesion with terminal aneurysmal dilatations at the edge of the AS near the macula with an associated BVN.

A diagnosis of PCV secondary to AS in the right eye was made. The patient was initiated on aflibercept injections in the right eye according to a treat-and-extend protocol. At this point, visual acuity was 20/40 in the right eye and 20/32 in the left eye. At 2 months' followup, the PED and PCV lesion had flattened (Fig. 3c). OCT (Fig. 3d) at 7 months showed an absence of SRF and PED, with complete regression of the polypoidal lesion. A total number of 15 injections were given in the right eye over 33 months with an average of 68 days between injections. In the first year, injections were given on average every 46 days, and in the fourth year, on average every 66 days, demonstrating the ability to extend treatment intervals.

At 53 months' follow-up, the patient experienced worsening of vision in the left eye, 29 months after the last ranibizumab injection. Fundoscopy revealed an orange nodule (Fig. 3i), and OCT examination demonstrated a PED and shallow SRF (Fig. 3j). FA (Fig. 3m, n) and ICGA (Fig. 3o, p) revealed early filling of a grape-like structure suggestive of PCV with leakage in the late phase and an associated small BVN in both the early and late phase. A diagnosis of PCV secondary to AS was made in the left eye. Intravitreal aflibercept injections were commenced in the left eye according to a treat-and-extend protocol based on the same visual acuity and OCT retreatment criteria detailed above. At 2 months after initiating aflibercept in the left eye, SRF was absent (Fig. 3k); however, the PED was still present. At the final followup, 26 months after aflibercept initiation (78 months following ranibizumab initiation), the PED was persisting; however, it had improved with the absence of SRF and intraretinal fluid (Fig. 31). Final best corrected visual acuity was 20/20 in the right eye and 20/32 in the left eye. Subsequent ICGA demonstrated involution of the PCV lesions in the left eye. A total number of 18 injections were given in the left eye with an average of 65 days between injections. In the first year, injections were given on average every 33 days, and in the fourth year, on average every 57 days. 


\section{Case Reports in Ophthalmology}

\section{Discussion}

Whilst CNV secondary to AS is commonly reported, PCV secondary to AS is often underdiagnosed for several reasons. PCV is often mistaken for occult (Type 1) CNV lesions, and its variable natural course further renders recognition difficult. Often ICGA, the gold standard for PCV diagnosis, is not performed or not available routinely in clinical practice despite being an important investigative tool. We hope to increase the awareness of PCV secondary to AS and thus improve screening rates for these clinically important illnesses.

It has previously been shown that in PCV patients who develop ranibizumab tachyphylaxis, switching therapy to aflibercept may maintain or improve visual acuity and regress polypoidal lesions without adverse events [7]. However, the current literature does not document the use of these treatments in cases of PCV secondary to AS. Here, we demonstrate that an inject-and-extend protocol with intravitreal aflibercept and ranibizumab is effective against PCV secondary to AS. Promisingly, visual acuity improved in all 3 cases and polypoidal lesions were stabilised using both anti-VEGF agents. In Case 1, the injection frequency in the fourth year with aflibercept was less than in the first year with ranibizumab; however, this may be reflective of the stability of the lesion rather than a difference in the efficacy between the 2 agents. A comparison between aflibercept and ranibizumab for PCV secondary to AS is beyond the scope of this report; however, it would represent an interesting study. Our cases were treated for 4 years; however, treatment may continue indefinitely in other patients reflecting the relapsing and remitting nature of PCV.

While AS is often asymptomatic, the presence of associated CNV or PCV may play a role in the choice of treatment for the patient. A previous study outlined a case of PCV secondary to AS in a symptomatic patient with PXE treated with verteporfin PDT combined with triamcinolone and the anti-VEGF agent bevacizumab [8]. However, this management strategy was suboptimal, as visual acuity in the affected eye deteriorated from 20/125 to 20/200 [8]. PDT has been associated with retinal toxicity, scarring, and atrophy, thus caution must be taken in cases with AS which suggest an inherently pathologically weak retina due to the structural dehiscences in Bruch's membrane [9]. However, it has been argued that a combination therapy using PDT and anti-VEGF agents may be effective in the treatment of typical PCV by combining mechanisms of angio-occlusion and anti-angiogenesis. Indeed the multicentre, double-masked EVEREST trial demonstrated a PDT-ranibizumab combination therapy to be more efficacious than an anti-VEGF monotherapy [10]. Thus, a PDT combination therapy was used in our Case 1 when ranibizumab monotherapy maintenance treatment failed.

Here, we describe the first cases reported of familial AS with PCV in a mother and son. AS are a hereditary condition (OMIM 607140); however, the inheritance pattern remains unidentified. Prompt recognition of AS is important due to its common associations with various, life-threatening, systemic diseases. In particular, the connective tissue disorder PXE may affect the gastrointestinal tract, great vessels, and arteries which may result in sudden death due to haemorrhage and accelerated atherosclerosis. Similarly, the early diagnosis of PCV is critical for optimizing treatment outcomes and prognosis. One natural history study demonstrated that without treatment for over 2 years, PCV patients may experience persistent polypoidal lesions, repeated haemorrhage and leakage, and significant vision loss [11]. In Case 2, the definitive diagnosis of PCV occurred following the long-term recurrence of a lesion initially diagnosed as typical CNV secondary to AS. PCV lesions have been known to arise secondarily to other established lesions including CNV [12]. Also, PCV is now recognised to be a variant of Type 1 CNV lesions, with the BVN and PCV components growing be- 
low the RPE within Bruch's membrane, often giving rise to the so-called "double-layer sign" on OCT [13]. We have previously described 2 cases of PCV secondary to choroidal nevus, where we hypothesised that possible chronic inflammatory changes may stimulate the growth of PCV. [14]. It is possible that in our current study the dehiscences in Bruch's membrane may give rise to a chronic inflammatory response also stimulating the development of PCV.

\section{Conclusion}

A treat-and-extend protocol with intravitreal aflibercept and ranibizumab with or without PDT for the treatment of PCV secondary to AS is safe and effective in the longer term. Our cases had improvement in vision and stabilisation of polyp lesions. We recommend the use of ICGA to assist in the diagnosis of PCV secondary to AS, since both conditions are underrecognised. Future studies are required to elucidate the underlying pathogenetic mechanisms in the development of PCV secondary to AS as well as optimise treatment for improved patient outcomes.

\section{Disclosure Statement}

The authors of this paper have no proprietary interest. None of the authors has any conflicts of interest related to this paper.

\section{References}

1 Clarkson JG, Altman RD: Angioid streaks. Surv Ophthalmol 1982;26:235-246.

-2 Hagedoorn A: Angioid streaks and traumatic ruptures of Bruch's membrane. Br J Ophthalmol 1975;59:267.

-3 Shields JA, Federman JL, Tomer TL, Annesley WH Jr: Angioid streaks. I. Ophthalmoscopic variations and diagnostic problems. Br J Ophthalmol 1975;59:257-266.

4 Dithmar S, Holz FG: Fluorescence Angiography in Ophthalmology. Heidelberg, Springer Medizin Verlag, 2008.

-5 Imamura Y, Engelbert M, Iida T, Freund KB, Yannuzzi LA: Polypoidal choroidal vasculopathy: a review. Surv Ophthalmol 2010;55:501-515.

-6 Koh AH, Expert PCVP, Chen LJ, Chen SJ, Chen Y, Giridhar A, Iida T, Kim H, Yuk Yau Lai T, Lee WK, Li X, Han Lim T, Ruamviboonsuk P, Sharma T, Tang S, Yuzawa M: Polypoidal choroidal vasculopathy: evidence-based guidelines for clinical diagnosis and treatment. Retina 2013;33:686-716.

7 Miura M, Iwasaki T, Goto H: Intravitreal aflibercept for polypoidal choroidal vasculopathy after developing ranibizumab tachyphylaxis. Clin Ophthalmol 2013;7:1591-1595.

8 Baillif-Gostoli S, Quaranta-El Maftouhi M, Mauget-Faysse M: Polypoidal choroidal vasculopathy in a patient with angioid streaks secondary to pseudoxanthoma elasticum. Graefes Arch Clin Exp Ophthalmol 2010;248:1845-1848.

-9 Duncan JL, Paskowitz DM, Nune GC, Yasumura D, Yang H, Matthes MT, Zarbin MA, LaVail MM: Retinal damage caused by photodynamic therapy can be reduced using BDNF. Adv Exp Med Biol 2006;572:297-302.

10 Koh A, Lee WK, Chen LJ, Chen SJ, Hashad Y, Kim H, Lai TY, Pilz S, Ruamviboonsuk P, Tokaji E, Weisberger A, Lim TH: EVEREST study: efficacy and safety of verteporfin photodynamic therapy in combination with ranibizumab or alone versus ranibizumab monotherapy in patients with symptomatic macular polypoidal choroidal vasculopathy. Retina 2012;32:1453-1464.

11 Bessho H, Honda S, Imai H, Negi A: Natural course and funduscopic findings of polypoidal choroidal vasculopathy in a Japanese population over 1 year of follow-up. Retina 2011;31:1598-1602. 
Wong and Qian: Long-Term Follow-Up of Polypoidal Choroidal Vasculopathy Secondary to Angioid Streaks Treated by Intravitreal Aflibercept and Ranibizumab

12 Otsuji T, Tsumura A, Takahashi K, Sho K, Nagai Y, Fukuchi T, Arisawa A, Matsumara M: Evaluation of cases of polypoidal choroidal vasculopathy showing classic choroidal neovascularization in their natural course. Nippon Ganka Gakkai Zasshi 2006;110:454-461.

13 St Martin JM, Rodman J, Pizzimenti JJ, Duchnowski E: The "double-layer sign": in vivo imaging of polypoidal choroidal vasculopathy. Optom Vis Sci 2013;90:e293-e300.

14 Wong JG, Lai XJ, Sarafian RY, Wong HS, Smith JB: Polypoidal choroidal vasculopathy secondary to a stable choroidal nevus. Retin Cases Brief Rep 2016;10:221-224. 


\section{Case Reports in Ophthalmology}

Case Rep Ophthalmol 2017;8:221-231 DOI: $10.1159 / 000468144$

(C) 2017 The Author(s). Published by S. Karger AG, Basel www.karger.com/cop

Wong and Qian: Long-Term Follow-Up of Polypoidal Choroidal Vasculopathy Secondary to Angioid Streaks Treated by Intravitreal Aflibercept and Ranibizumab
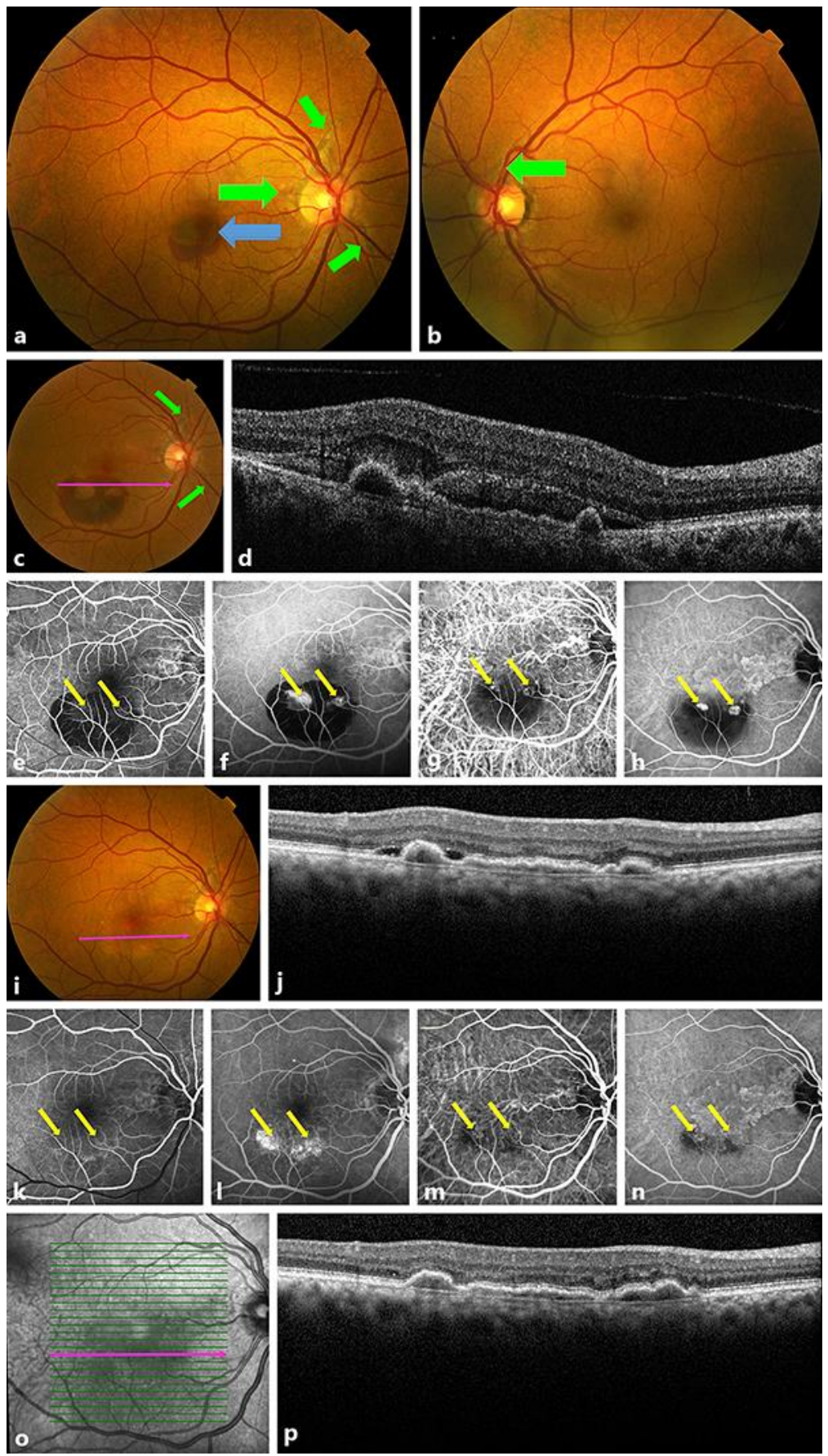


\section{Case Reports in Ophthalmology}

Fig. 1. a, b Colour fundus photography of Case 1 demonstrating bilateral angioid streaks (AS, green arrows), macular subretinal haemorrhage, and retinal pigment epithelial detachment (PED) (blue arrow). Case 1 at 3 years following the initiation of treatment. c Colour fundus photography demonstrates AS in the right eye (green arrows), with recurrence of a large subretinal haemorrhage, subretinal fluid (SRF), and PED. d OCT scan reveals subretinal haemorrhage, SRF, and PED. Fluorescein angiography (FA) demonstrates early filling of 2 grape-like polypoidal structures (e) with leakage in the late phase at the inferior region of the macula (f), corresponding to polypoidal choroidal vasculopathy (PCV) lesions at the edge of the AS (yellow arrows). Indocyanine green angiography (ICGA) also demonstrates early filling (g) and leakage in the late phase of the PCV lesions (yellow arrows) (h), as well as an associated branching vascular network (BVN) in both early and late phase. At 20 months after this visit, fundoscopy (i) and OCT (j) showed small SRF and PED with no subretinal haemorrhage; FA (k) and ICGA (m) in the early phase still revealed the PCV lesions and hyperfluorescence in the late phase in FA (I) and ICGA (yellow arrows) (n). The patient was then switched to intravitreal aflibercept, and at 27 months after the initial aflibercept injection, the OCT scan in $\mathbf{p}$ shows a small PED, and an absence of subretinal haemorrhage and SRF. Thin purple arrows in $\mathbf{c}, \mathbf{i}$, and $\mathbf{o}$ demonstrate the locations of the cross-sectional slides of the OCT images in $\mathbf{d}, \mathbf{j}$, and $\mathbf{p}$, respectively.
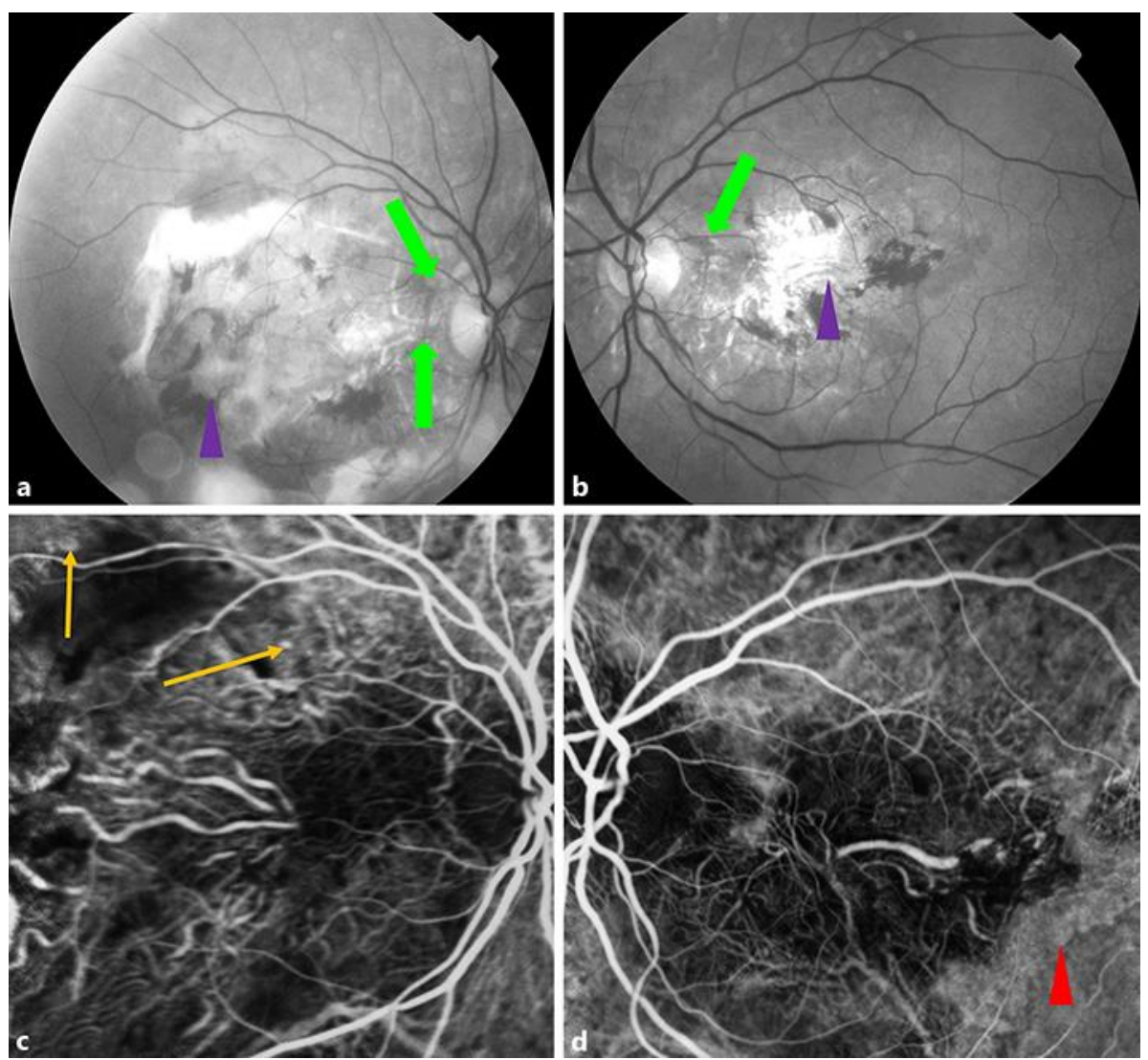

Fig. 2. Red free fundus photos (a, b) of Case 2 (mother of Case 1) demonstrating bilateral angioid streaks (thick green arrows) and fibrotic disciform scars (purple triangles). Indocyanine green angiography (c, d) demonstrates polyp lesions at the edge of the disciform scars in the right eye (yellow arrow) and late geographic hypercyanescence in the left eye (red triangle) demonstrating the appearance of a branching vascular network, consistent with a diagnosis of advanced polypoidal choroidal vasculopathy. 


\section{Case Reports in Ophthalmology}

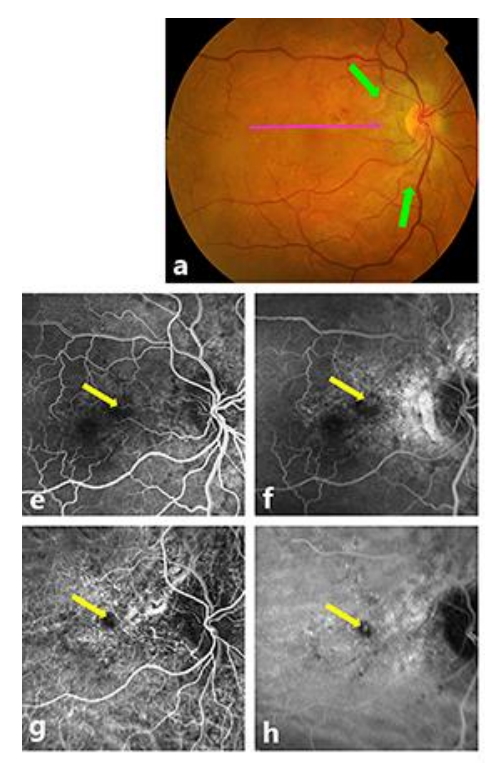

(C) 2017 The Author(s). Published by S. Karger AG, Basel www.karger.com/cop

Wong and Qian: Long-Term Follow-Up of Polypoidal Choroidal Vasculopathy Secondary to Angioid Streaks Treated by Intravitreal Aflibercept and Ranibizumab
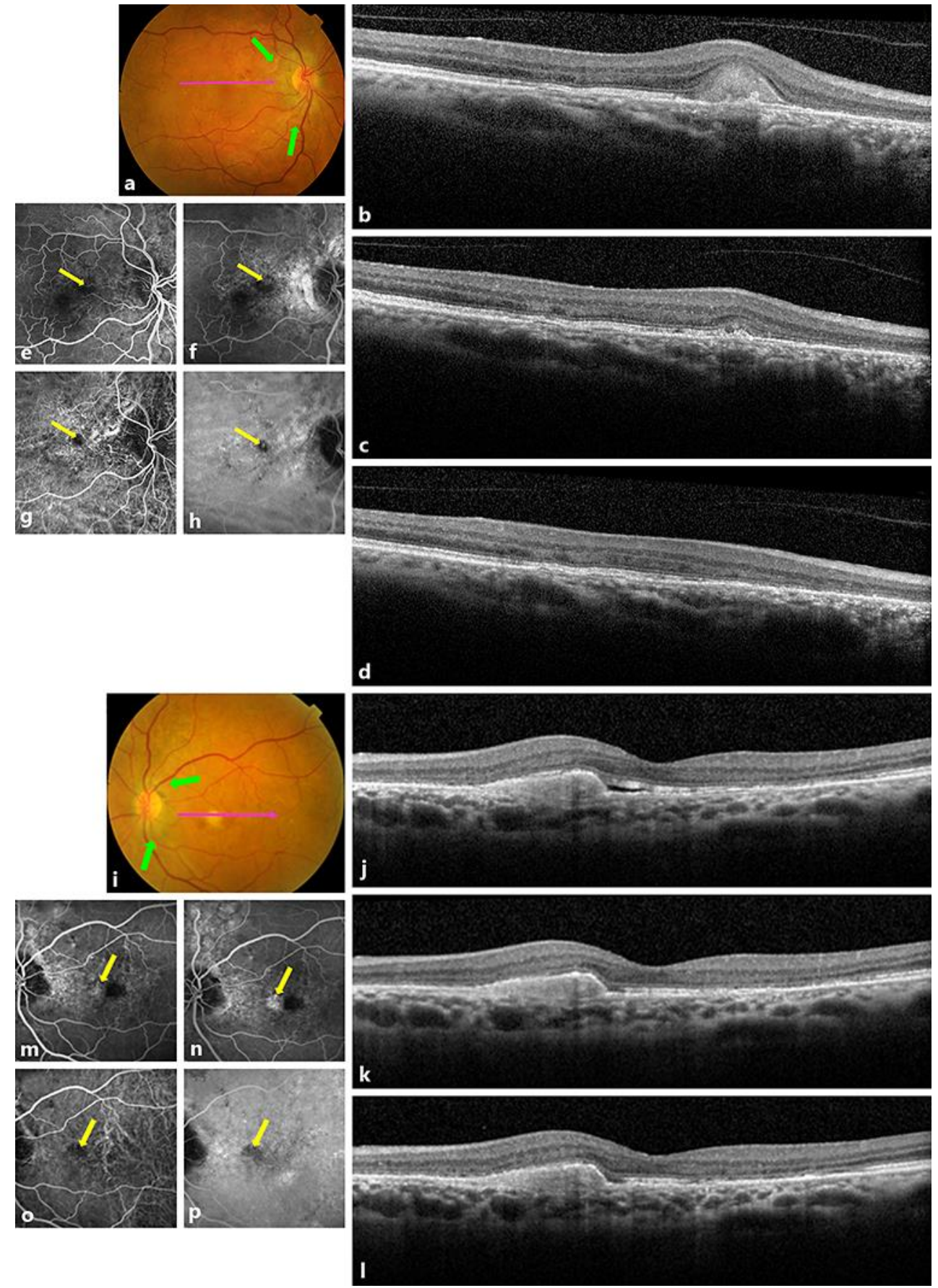
Fig. 3. Case 3 at 3 years following treatment initiation. a Colour fundus photography demonstrates a discreet orange-red nodule in the nasal macula connecting to the angioid streaks (AS) in the right eye (green arrow; as described in Fig. 2A); b OCT reveals a PED with an underlying polypoidal lesion, with no subretinal or intraretinal fluid; fluorescein angiography (FA) in the early (e) and late phase (f) and ICGA in the early (g) and late phase (h) also reveal a grape-like polypoidal structure with leakage at late phase on the nasal aspect of the macula, corresponding to a PCV lesion secondary to the AS (yellow arrow). The patient was then switched to intravitreal aflibercept and the OCT scan at 2 months after the initial aflibercept injection revealed regression of the PCV lesion (c); the OCT scan at 7 months showed an absence of SRF and PED (d). The thin purple arrow in a demonstrates the locations of the cross-sectional slides of the OCT images in b, c, and d. Case 3 at the 3.5-year follow-up visit in the left eye. i Colour fundus photography demonstrates an orange-yellow nodule at the nasal edge of the macula and adjacent to the AS in the left eye (thick green arrow; as described in c), corresponding to a PCV lesion; $\mathbf{j}$ the OCT scan showed a PED associated with an underlying discreet polyp-like lesion and a small SRF next to the lesion; FA (m) and ICGA (n) demonstrate early filling of a grape-like structure suggestive of PCV, with leakage in the late phase in FA (o) and ICGA (p) (yellow arrow). OCT revealed a persisting PED with an underlying polypoidal lesion and the absence of SRF at 2 months (k) and 20 months (I) after this visit. The thin purple arrow in $\mathbf{i}$ demonstrates the locations of the cross-sectional slides of the OCT images in $\mathbf{j}, \mathbf{k}$, and $\mathbf{I}$. 